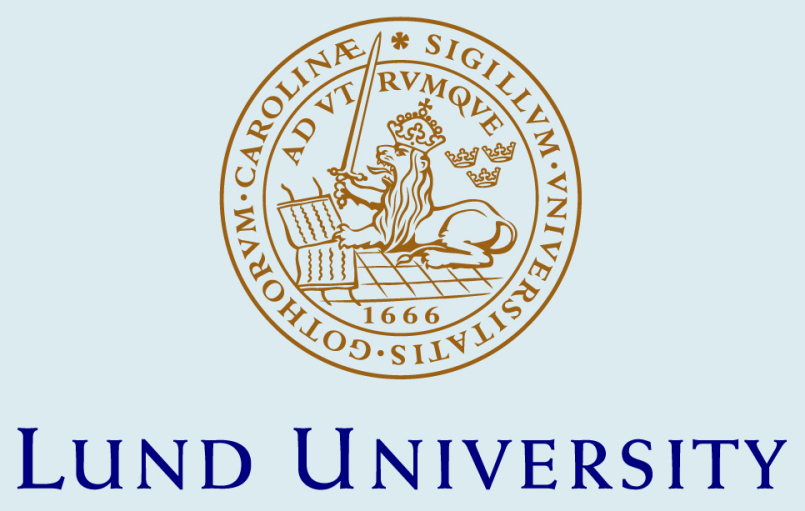

\title{
Distributed Low-Complexity Controller for Wind Power Plant in Derated Operation
}

\author{
Biegel, Benjamin; Madjidian, Daria; Spudic, Vedrana; Rantzer, Anders; Stoustrup, Jakob
}

2013

Link to publication

Citation for published version (APA):

Biegel, B., Madjidian, D., Spudic, V., Rantzer, A., \& Stoustrup, J. (2013). Distributed Low-Complexity Controller for Wind Power Plant in Derated Operation. Paper presented at IEEE Multi-conference on Systems and Control, 2013, Hyderabad, India.

Total number of authors:

5

\section{General rights}

Unless other specific re-use rights are stated the following general rights apply:

Copyright and moral rights for the publications made accessible in the public portal are retained by the authors

and/or other copyright owners and it is a condition of accessing publications that users recognise and abide by the

legal requirements associated with these rights.

- Users may download and print one copy of any publication from the public portal for the purpose of private study or research.

- You may not further distribute the material or use it for any profit-making activity or commercial gain

- You may freely distribute the URL identifying the publication in the public portal

Read more about Creative commons licenses: https://creativecommons.org/licenses/

Take down policy

If you believe that this document breaches copyright please contact us providing details, and we will remove access to the work immediately and investigate your claim. 


\title{
Distributed Low-Complexity Controller for Wind Power Plant in Derated Operation
}

\author{
Benjamin Biegel Daria Madjidian Vedrana Spudić Anders Rantzer Jakob Stoustrup
}

\begin{abstract}
We consider a wind power plant of megawatt wind turbines operating in derated mode. When operating in this mode, the wind power plant controller is free to distribute power set-points to the individual turbines, as long as the total power demand is met. In this work, we design a controller that exploits this freedom to reduce the fatigue on the turbines in the wind power plant. We show that the controller can be designed in a decentralized manner, such that each wind turbine is equipped with a local low-complexity controller relying only on few measurements and little communication.

As a basis for the controller design, a linear wind turbine model is constructed and verified in an operational wind power plant of megawatt turbines. Due to limitations of the wind power plant available for tests, it is not possible to implement the developed controller; instead the final distributed controller is evaluated via simulations using an industrial wind turbine model. The simulations consistently show fatigue reductions in the magnitude of $15-20 \%$.
\end{abstract}

\section{INTRODUCTION}

The full energy production of wind turbines is not always valuable: in Denmark the wind production occasionally covers more than $100 \%$ of the energy demand [1] forcing Denmark to export the excess energy to neighboring countries at low prices, possibly even negative prices [2]. The difficulties in integrating wind energy are expected to increase in the following years as the wind penetration will increase: during the last 30 years, the wind penetration in Denmark has increased from around $0 \%$ to approximately $25 \%$ today while it is expected that the 2020 penetration is $49.5 \%$ [3].

In addition to the issue of overproduction, the need for ancillary services are expected to increase [4]. One approach towards resolving the future overproduction issues and increased needs for ancillary services is the smart grid concept, where consumers take part in the balancing effort (see, e.g., [5], [6], [7], [8]). A second approach suitable in combination with the smart grid solution, is to reduce the wind turbine power production, leaving a margin (or delta) between the available wind energy and the produced energy. A wind power plant with all turbines running in such delta mode is able to offer regulating power to the system operator.

B. Biegel and J. Stoustrup are with the Department of Electronic Systems, Automation and Control, Aalborg University, Denmark; e-mail: $\{$ bbi, jakob\}@es.aau.dk.

D. Madjidian and A. Rantzer are with the Department of Automatic Control, Lund University, Sweden; e-mail: \{daria, Anders.Rantzer\}@control.lth.se.

V. Spudić is with the Department of Control and Computer Engineering, Faculty of Electrical Engineering and Computing, University of Zagreb, Croatia; vedrana.spudic@ fer.hr

The work is under the European research project Aeolus funded by the European Commission.
In the electricity market context, this could be realized by bidding wind energy into the reserve market.

The use of wind power plants in the energy reserve market is beneficial from multiple perspectives. Recent reports suggest that wind turbines are well suited to provide frequency regulating services as the primary response from wind power is faster than those from conventional generation [9], [10]. Further, [11] describes that when moving towards energy systems of high penetration of renewable energy, the need of energy storage can be significantly reduced by allowing a certain amount of overcapacity of the renewable sources.

In this work, we consider the case where a wind power plant profits from selling regulating services and therefore produces less power than available, i.e., the turbines are in derated operation. With more power available than demanded, the wind power plant controller is free to distribute the power production among the turbines, as long as the power demand is met. This freedom is not exploited in current wind power plants, as the power distribution is done in a static manner based on long term measurements and predictions [12], [13].

We develop a controller that exploits this power distribution freedom to vary the power set-points of the turbines dynamically in response to the local wind conditions, such that the total fatigue, or the fatigue of certain turbines, is minimized. Similar strategies are presented in [14] and [15]. Reducing the fatigue is beneficial as it allows a cheaper wind turbine design, for example by requiring less steel for the tower, or longer turbine operation time and possibly a reduced need for maintenance. Another application is to use the wind power plant controller to reduce the fatigue loading of certain exposed turbines while allowing other turbines to experience a higher fatigue load such that the wind turbines are worn out at the same time.

Both a centralized and distributed controller are designed. The centralized controller requires state information from all turbines in the wind power plant to determine the power set-point of each turbine. The distributed controller allows a simple and transparent design where each wind turbine contains a part of the wind power plant controller and is able to update its own power set-point relying only on very limited communication and computation effort.

As a basis for controller design, a linear model of a closed loop megawatt turbine with effective wind speed and power set-point as inputs is used. The closed loop turbine model is verified via several sequences of real life experiments on 8 neighboring megawatt turbines in an operating wind power plant. The turbines are excited by applying power set-point 


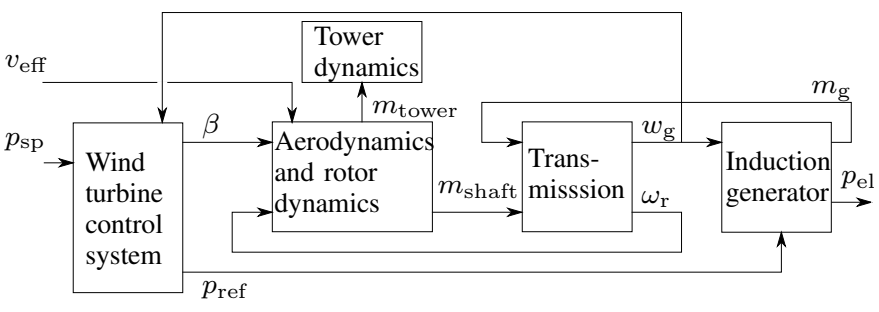

Fig. 1. Variable speed pitch controlled wind turbine block diagram.

updates and the linear model is verified by comparing turbine measurements with the corresponding state trajectories of the linear model.

The outline of the rest of the paper is as follows. First, in Section II we describe the megawatt wind turbine and the linear closed loop model. Next, in Section III we present the validation results of the model based on wind power plant measurements. Following, in Section IV, the main results are presented: a centralized and a distributed wind power plant controller designed to minimize or shift fatigue on the turbines in the wind power plant. Section V presents simulation examples that illustrate the benefits of the design, and finally Section VI sums up the work.

\section{Megawatt Wind Turbine Model}

The basis of this work is a $3 \mathrm{MW}$ horizontal axis, variable speed, pitch regulated wind turbine that is currently widely used. In the following, we describe this wind turbine at an overall level. Following, we present a linearized turbine model where the inputs are the power set-point and effective wind speed. This linearized model is verified in Section III via real life experiments conducted in a megawatt wind power plant.

\section{A. Overall Model}

Due to the competitive nature of the wind turbine industry and resulting confidentiality issues, the modeling is kept at a conceptual level. For more detailed modeling of pitch regulated wind turbines see, e.g., [16], [17] or [18].

Fig. 1 shows the main subsystems of the wind turbine. The incoming effective wind speed $v_{\text {eff }}$ generates a torque on the rotor shaft denoted $m_{\text {shaft }}$ causing the rotor to rotate with angular velocity $\omega_{\mathrm{r}}$. Further, the incoming wind generates a torque on the tower, $m_{\text {tower. }}$ Through a gearbox, the torque $m_{\text {shaft }}$ on the rotor shaft drives a doubly-fed induction generator to rotate with velocity $\omega_{\mathrm{g}}$. The power production $p_{\mathrm{el}}$ of the induction generator is controllable through the generator torque $m_{\mathrm{g}}$. The power set-point $p_{\mathrm{sp}}$ to the turbine is handled by the wind turbine control system: by controlling the blade pitch angle $\beta$ and the generator torque via a power reference $p_{\text {ref }}$, the control system maintains operation at the desired rotor angular velocity $\omega_{r}$ while keeping $p_{\mathrm{el}}$ close to $p_{\mathrm{sp}}$.

\section{B. Linearized Model}

A linear discrete-time model is developed as a basis for the wind power plant controller design. This model is verified via real life measurements obtained in a wind power plant of megawatt wind turbines. Due to the sampling limitations of the wind farm communication network, the linear discrete time model is designed using a sampling rate of 1 second. With this sampling rate, only the slowest dynamics of the wind turbine are modeled thus omitting faster phenomena including shaft and tower dynamics. Such simplified modeling may seem restrictive in light of the fatigue load reduction objective; however, it should be noted that the aim is to develop a wind power plant control strategy based on power set-point distribution. This means that every wind turbine contains its own local controller that handles the wind turbine structural responses, while the wind power plant controller reduces the excitation of the turbine structure via power control. Note, however, that the presented scheme could easily be extended to include more dynamics if the given wind farm communication network allows faster sampling.

The wind power plant control signals are the power setpoints to the turbines in the wind power plant. A power setpoint can be tracked by the wind turbine if the available power is larger than the power set-point; if this is not the case, the wind turbine controller switches to power maximization and the power set-point no longer influences wind turbine operation. In this work we only consider the operating regime where there is more power available than demanded at all turbines. At a time scale of seconds, the relevant dynamics in this regime arise from the rotor inertia and the wind turbine pitch control system. The main source of nonlinearities is the rotor aerodynamics.

The linearized wind turbine model takes the effective wind speed $v_{\text {eff }}$ and the power set-point $p_{\text {sp }}$ as inputs, the states are the pitch angle $\beta$ and the generator velocity $w_{\mathrm{g}}$, while the outputs are the tower torque $m_{\text {tower }}$ and shaft torque $m_{\text {shaft }}$. The small signal values of the states, inputs and outputs for turbine $i$ are modeled by the second order state space model

$$
\begin{array}{ll}
x_{i}(k+1) & =A_{i} x_{i}(k)+B_{1 i} u_{i}(k)+B_{2 i} w_{i}(k) \\
y_{i}(k) & =C_{i} x_{i}(k)+D_{1 i} u_{i}(k)+D_{2 i} w_{i}(k),
\end{array}
$$

where

$$
\begin{aligned}
u_{i} & =p_{\mathrm{sp}, i}-\bar{p}_{\mathrm{sp}, i} \in \mathbf{R}, \\
x_{i} & =\left(\beta_{i}-\bar{\beta}, \omega_{\mathrm{r}, i}-\bar{\omega}_{\mathrm{r}, i}\right)^{T} \in \mathbf{R}^{2}, \\
w_{i} & =v_{\mathrm{eff}, i}-\bar{v}_{\mathrm{eff}, i} \in \mathbf{R}, \\
y_{i} & =\left(m_{\mathrm{tower}, i}-\bar{m}_{\mathrm{tower}, i}, m_{\mathrm{shaft}, i}-\bar{m}_{\mathrm{shaft}, i}\right)^{T} \in \mathbf{R}^{2},
\end{aligned}
$$

and $\left(\bar{p}_{\mathrm{sp}, i}, \bar{\beta}_{i}, \bar{\omega}_{r, i}, \bar{v}_{\mathrm{eff}, i}, \bar{m}_{\mathrm{shaft}, i}, \bar{m}_{\mathrm{shaft}, i}\right)$ is the operating point of turbine $i$. It can further be assumed that

$$
p_{\mathrm{el}, i}(k)=p_{\mathrm{sp}, i}(k),
$$

as the dynamics of the electrical subsystem, that govern the power regulation, are much faster than the given sampling time of $1 \mathrm{~Hz}$. A more thorough description of the model simplifications and linearization is found in [19].

\section{Model Validation}

We validate the linear megawatt turbine model by conducting experiments at an operational wind power plant. In the experiments, sequences of steps in the power set-points are 
applied to a megawatt turbine while measurements are made of point wind speed, pitch angle, rotor speed, generator speed and electrical power output. Based on these measurements, the effective wind speed $v_{\text {eff }}$ is estimated using the extended Kalman filter based tool described in [20].

By taking the observed effective wind speed and power set-points as inputs to the linear model, we are able to compare the linear model with the measurements from the operational turbines. The model validation shows strong correlation between the model and the observed measurements. The experiment was repeated 18 times in two different operating regions with similar results. The normalized correlation between measurements and the linear model is consistent: around 0.95 for electrical power, 0.95 for pitch angle and 0.50 for the generator speed. It is the authors' belief that the discrepancies in the generator speed model are due to the approximation of the effective wind speed. Otherwise, the signals are similar leading to the conclusion that the linear model is satisfactory for wind power plant control purposes.

\section{CONTROller Synthesis}

In the following we construct a wind power plant model which we use as a basis for controller design.

\section{A. Wind Power Plant Model}

The power demand to the wind power plant is denoted $p_{\mathrm{wpp}}$ and is achieved by distributing operating points $\bar{p}_{\mathrm{sp}}$ such that

$$
\sum_{i=1}^{n} \bar{p}_{\mathrm{sp}, i}=p_{\mathrm{wpp}}
$$

where $n$ is the number of turbines in the wind power plant. In the wind power plant model we only consider the coupling of the wind turbines through the power constraint (3).

For a number of reasons, it is chosen to neglect the coupling of the wind turbines through the wind field. First, a coupling model would need to contain a delay element in order to account for the time it takes for the wind to travel from one turbine to another. Including coupling in the model would therefore add significantly to the complexity of the problem and result in a high order controller. Second, there is a lack of reliable models describing how effective wind speed variations at neighboring turbines are related. Third, although we agree that coupling is very important when considering static models (models describing mean wind speed, turbulence level, mean power production, etc.), we believe it is less important when dealing with variations around these mean levels as in this work. Finally, the controller designed in this work will only apply very small changes in the power set-point of the turbines resulting in small pitch perturbation; evidently, the effect such small perturbations in pitch will disperse in the natural wind variations before it reaches the neighboring turbines several hundred meters downstream.

Most importantly, while we neglect the coupling in the controller design, the coupling is indeed included in the controller evaluation, as discussed later. We would like to emphasize that even though the controllers were designed without taking coupling into account, they perform well in a simulation environment where coupling is present.

1) Dynamics: We describe a wind power plant consisting of $n$ wind turbines as

$$
\begin{array}{ll}
x(k+1) & =A x(k)+B_{1} u(k)+B_{2} w(k) \\
y(k) & =C x(k)+D_{1} u(k)+D_{2} w(k)
\end{array}
$$

where $x, y \in \mathbf{R}^{2 n}, u, w \in \mathbf{R}^{n}$ are obtained by stacking the inputs, states and outputs horizontally, and $B_{1}, B_{2}, D_{1}, D_{2} \in$ $\mathbf{R}^{2 n \times n}, A, C \in \mathbf{R}^{2 n \times 2 n}$ are obtained by forming the system matrices block diagonally, e.g.,

$$
\begin{aligned}
& x=\left(x_{1}^{T}, \ldots, x_{n}^{T}\right)^{T} \in \mathbf{R}^{2 n} \\
& A=\operatorname{diag}\left(A_{i}, \ldots, A_{n}\right) \in \mathbf{R}^{2 n \times 2 n}
\end{aligned}
$$

and similarly for the remaining vectors and matrices.

Each turbine in the wind power plant is free to vary its power set-point $p_{\mathrm{sp}}$ around its operating point $\bar{p}_{\mathrm{sp}}$ as long as the variations of the turbines sum to zero; in this way we assure that the demand $p_{\mathrm{wpp}}$ is met. We achieve this by requiring that

$$
\mathbf{1}^{T} u(k)=0
$$

where $\mathbf{1}$ is a vector of all ones.

2) Objective: The objective is to minimize the fatigue experienced by the turbines int he wind power plant. The literature suggests that the variance in tower bending torque and shaft torsion torque can be used as a measure of fatigue, see e.g. [21], [14]. Further, the power set-points $p_{\mathrm{sp}}$ must be kept close to the operating point where the linear model is valid. We therefore formulate the wind power plant objective as follows:

$$
\ell(y, u)=\mathbf{E}\left(y^{T} Q y+u^{T} R u\right)
$$

where $Q \in \mathbf{R}^{2 n \times 2 n}, R \in \mathbf{R}^{n \times n}$ are diagonal matrices penalizing fatigue and power set-point variations of the $n$ wind turbines. Note that this setup allows us to penalize the fatigue on certain wind turbines more than others by selecting different diagonal values $Q_{i i}$, enabling us able to shift load between the turbines.

\section{B. Problem Formulation}

Based on the wind power plant objective and dynamics, we formulate the control problem as follows.

$$
\begin{array}{ll}
\text { minimize } & \mathbf{E}\left(y^{T} Q y+u^{T} R u\right) \\
\text { subject to } & x(k+1)=A x(k)+B_{1} u(k)+B_{2} w(k) \\
& y(k)=C x(k)+D_{1} u(k)+D_{2} w(k) \\
& \mathbf{1}^{T} u(k)=0, k=0,1, \ldots
\end{array}
$$

with variables $x(k), y(k) \in \mathbf{R}^{2 n}, u(k) \in \mathbf{R}^{n}$. We eliminate the power constraint $\mathbf{1}^{T} u=0$ by finding a matrix $T \in$ $\mathbf{R}^{n \times n-1}$ that parameterizes the linear feasible set [22]:

$$
\left\{u \in \mathbf{R}^{n} \mid \mathbf{1}^{T} u=0\right\}=\left\{T \hat{u} \mid \hat{u} \in \mathbf{R}^{n-1}\right\} .
$$

We choose $T$ to have all elements on the main diagonal equal 1 and the first lower subdiagonal equal -1 , the rest of 
the entries are 0 . Hereby we obtain $u=T \hat{u}$ which can be written as

$$
\begin{aligned}
& u_{1}=\hat{u}_{1} \\
& u_{i}=\hat{u}_{i}-\hat{u}_{i-1}, \quad i=1, \ldots, n-1 \\
& u_{n}=-\hat{u}_{n-1} .
\end{aligned}
$$

Problem (8) can now be stated as

$$
\begin{array}{ll}
\text { minimize } & \mathbf{E}\left(y^{T} Q y+\hat{u}^{T} \hat{R} \hat{u}\right) \\
\text { subject to } & x(k+1)=A x(k)+\hat{B}_{1} \hat{u}(k)+B_{2} w(k) \\
& y(k)=C x(k)+\hat{D}_{1} \hat{u}(k)+D_{2} w(k) \\
& k=0,1, \ldots
\end{array}
$$

with variables $x(k), y(k) \in \mathbf{R}^{2 n}, \hat{u}(k) \in \mathbf{R}^{n-1}$ and where $\hat{R}=T^{T} R T, \hat{B}_{1}=T B_{1}, \hat{D}_{1}=T D_{1}$.

\section{Centralized Controller}

To keep the controller as simple as possible, we make the rough assumption that the incoming effective wind speed variations $w$ are white Gaussian noise. With this assumption, Problem 11 is a standard linear quadratic Gaussian problem where the solution is a state feedback law [23]

$$
\hat{u}(k)=-\hat{L}^{\star} x(k)
$$

where $\hat{L}^{\star} \in \mathbf{R}^{n-1 \times 2 n}$ is a time invariant feedback matrix. In terms of the original problem, the control law becomes

$$
u(k)=-L^{\star} x(k)
$$

where $L^{\star}=T \hat{L}^{\star} \in \mathbf{R}^{n \times 2 n}$.

1) Algorithm: Using the found optimal controller $L^{\star}$, the control algorithm can be stated. The pitch angles $\beta_{i}$ and the generator velocities $\omega_{\mathrm{g}, i}$ are measurable with high accuracy and we can use them directly in the controller algorithm: for $k=1,2, \ldots$.

1) Measure $\beta_{i}(k), \omega_{\mathrm{g}, i}(k)$ for all turbines $i=1, \ldots, n$ and construct $x(k)$ by subtracting the operating points.

2) Calculate the power set-point deviations $u(k)$ by the control law $u(k)=-L^{\star} x(k)$.

3) Provide new power set-points $p_{\mathrm{sp}}(k)=\bar{p}_{\mathrm{sp}}(k)+u(k)$ to the $n$ turbines.

\section{Distributed Controller}

In the distributed control scheme, each wind turbine is only allowed to communicate with certain other wind turbines. The communication structure can be chosen in any desired way and does not depend on the geographical positioning of the wind turbines as the coupling through the wind field is neglected. Based on the state feedback law $u(k)=-L x(k)$, we can formulate the distributed controller by imposing a structural restriction on $L$

$$
L \in \mathcal{L}
$$

where the set $\mathcal{L}$ defines the allowed communication structure. As an example of a communication structure, we assume that the wind turbines are arranged in a row position and only are allowed to communicate with the $m$ closest neighboring turbines at each side. This is illustrated in Fig. 2 (with $m=$

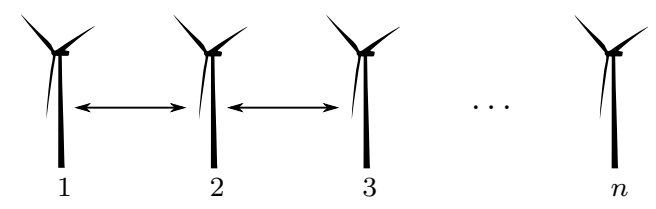

Fig. 2. Example of a wind power plant communication structure where the turbines are placed in a row formation and are only allowed to communicate with the closest neighbor on each side.

1). With this setup, the structural restriction can be described as

$$
\mathcal{L}=\left\{X \mid(X)_{i j} \neq 0 \text { only if } i-m \leq j \leq i+m\right\}
$$

where $(X)_{i j}$ is defined as block $(i, j)$ of $X$.

The structural constraints of $L$ imposes structural constraints on $\hat{L}$ as $L=T \hat{L}$. We denote this structural constraint

$$
\hat{L} \in \hat{\mathcal{L}} \text {. }
$$

Due to the chosen structure of the transformation matrix $T$, the structure $\hat{\mathcal{L}}$ is given by

$$
\hat{\mathcal{L}}=\left\{X \mid(X)_{i j} \neq 0 \text { only if } i-m+1 \leq j \leq i+m\right\} .
$$

For every stabilizing feedback on the form $\hat{u}(k)=-\hat{L} x(k)$ applied to Problem 11, define the cost $J(\hat{L})$ as

$$
J(\hat{L})=\mathbf{E}\left(y^{T} Q y+u^{T} \hat{R} u\right) .
$$

From [24] we know that the gradient of the cost with respect to $\hat{L}$ is given by

$$
\nabla J_{\hat{L}}=2\left(\left(\hat{R}+\hat{D}_{1}^{T} Q \hat{D}_{1}\right) \hat{L}-\hat{S}^{T}-\hat{B}_{1}^{T} P A_{\hat{L}}\right) X
$$

where $P$ and $X$ are the solutions to the Lyapunov equations

$$
\begin{aligned}
X= & A_{\hat{L}} X A_{\hat{L}}^{T}+B_{2}^{T} B_{2} W \\
P= & A_{\hat{L}}^{T} P A_{\hat{L}}+C^{T} Q C-\hat{S} \hat{L}-(\hat{S} \hat{L})^{T} \\
& +\hat{L}^{T}\left(\hat{R}+\hat{D}_{1}^{T} Q \hat{D}_{1}\right) \hat{L}
\end{aligned}
$$

where $W=\mathbf{E}\left(w w^{T}\right)$ and with $A_{\hat{L}}$ and $\hat{S}$ given by

$$
\begin{aligned}
& A_{\hat{L}}=A-\hat{B}_{1} \hat{L} \\
& \hat{S}=\frac{1}{2}\left(C^{T} Q \hat{D}_{1}+C^{T} Q^{T} \hat{D}_{1}\right) .
\end{aligned}
$$

To find a suboptimal feedback matrix $\hat{L}^{*} \in \hat{\mathcal{L}}$ we apply the gradient descent method as follows.

repeat

1) Find the negative gradient $-\nabla J_{\hat{L}}$ using (19).

2) Set the search descent direction equal to the negative gradient projected onto the structure $\hat{\mathcal{L}}$

$$
(\Delta \hat{L})_{i j}:= \begin{cases}-\left(\nabla J_{\hat{L}}\right)_{i j} & \text { if } i-m+1 \leq j \leq i+m . \\ 0 & \text { otherwise. }\end{cases}
$$

3) Choose a step length $\alpha$ based on some standard method.

4) Update the feedback matrix $\hat{L}:=\hat{L}+\alpha \Delta \hat{L}$

until some stopping criterion.

The final sparse feedback matrix denoted $\hat{L}^{*}$ is readily transformed back to the original structure $L^{*}=T \hat{L}^{*}$ which 
will obey the desired sparsity constraint $L^{*} \in \mathcal{L}$. Note that this algorithm is performed offline to obtain a desired feedback matrix, which then will be used online.

We use the verified linear wind turbine model as a basis for a distributed wind power plant controller design. We design the controller for a row of 8 wind turbines operating at wind speeds $15 \mathrm{~m} / \mathrm{s}$ producing 2.2 MW. Each turbine is allowed to communicate with $m$ neighboring turbines in each direction. The convergences of the sparse feedback matrices are illustrated in Fig. 3 for $m=1,2,3$ compared to the optimal central feedback matrix $L^{\star}$ and open loop $L=0$.

It is noticed that with $m \geq 2$ we get performance close to optimal. This means that we can distribute the controller to the turbines in the wind power plant and only loose little performance compared to full state feedback. The advantage is that this allows a low-complexity transparent design where each turbine only requires state information from the two closest neighboring turbines on each side to update its own power set-point.

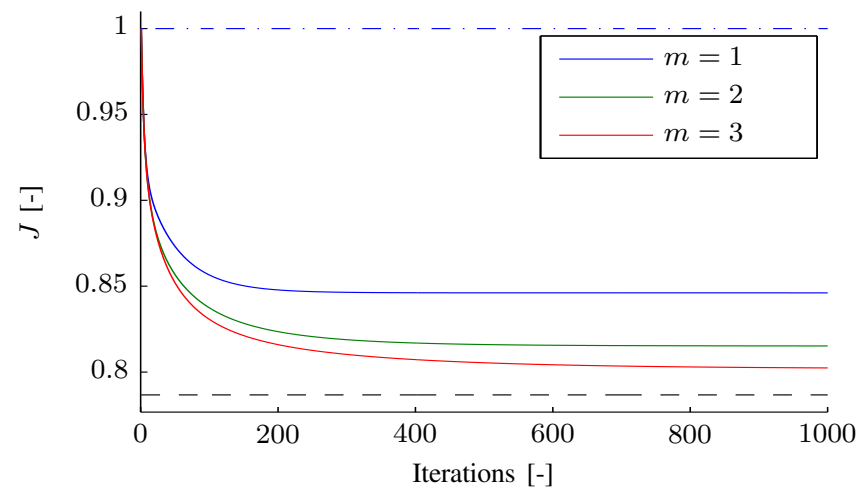

Fig. 3. Convergence of the feedback controller compared to open loop (blue, dot-dashed) and to optimal full state feedback (black, dashed).

\section{E. Algorithm}

Once a feedback matrix $L^{*}$ is found, we can form the distributed controller algorithm for turbine $i$.

for $k=1,2, \ldots$

1) Measure $\beta_{i}(k), \omega_{\mathrm{g}, i}(k)$ and form the local state $x_{i}(k)$.

2) Obtain $\tilde{x}_{i}(k)=\left[x_{i-m}^{T}(k), \ldots, x^{T}(k)_{i+m}\right]^{T} \in \mathbf{R}^{4 m+2}$ by communication with the $m$ neighboring turbines at each side.

3) Calculate the local power set-point deviation $u_{i}(k)=$ $L_{i}^{*} \tilde{x}_{i}(k)$

4) Apply the new local set-point $p_{\mathrm{sp}, i}(k)=\bar{p}_{\mathrm{sp}, i}+u_{i}(k)$. In the above, $L_{i}^{*} \in \mathbf{R}^{1 \times 4 m+2}$ is the block of $L^{*}$ corresponding to turbine $i$. This controller is running at all $n$ turbines.

\section{Controller Evaluation}

In this section we evaluate the distributed controller. The centralized controller reveals similar results but these results are not presented here. As the wind power plant available for tests does not support updates of power set-points every second, we are not able to implement the controller. Instead we verify the controller via simulations using sequences of

\begin{tabular}{|l|c|c|c|c|c|}
\hline Wind sequence & 1 & 2 & 3 & 4 & 5 \\
\hline \hline Tower fat. red. (linear) & $17 \%$ & $17 \%$ & $15 \%$ & $17 \%$ & $18 \%$ \\
Shaft fat. red. (linear) & $17 \%$ & $16 \%$ & $21 \%$ & $17 \%$ & $5 \%$ \\
\hline \hline Tower fat. red. (nonlinear) & $10 \%$ & $8 \%$ & $6 \%$ & $11 \%$ & $10 \%$ \\
Shaft fat. red. (nonlinear) & $29 \%$ & $25 \%$ & $29 \%$ & $29 \%$ & $17 \%$ \\
\hline
\end{tabular}

TABLE I

TOWER AND SHAFT FATIGUE REDUCTIONS OBSERVED IN THE LINEAR AND THE NONLINEAR WIND POWER PLANT SIMULATION, RESPECTIVELY.

effective wind speeds obtained from the experiments of the 8 neighboring megawatt turbines.

To provide as realistic a simulation environment as possible, the wind speeds used in all simulations are effective wind speed estimates based on measurements from Thanet offshore wind farm. It is important to notice that coupling hereby is included in the wind power plant controller evaluation as the effective wind speed estimates are gathered from real life neighboring wind turbines.

We consider the case where the available power exceeds the nominal $3 \mathrm{MW}$ of each of the 8 turbines. The wind power plant is demanded to produce $17.6 \mathrm{MW}$; the remaining 6.4 MW are sold as regulating power reserve. In the following, we first use the verified linear model for controller evaluation. Secondly, we use an industrial model as simulation platform to further enhance the credibility of the controller. In both simulations we allow communication with the $m=2$ closest neighboring turbines on each side. The reference is a controller that distributes the power demand equally among the turbines.

\section{A. Evaluation on Verified Linear Model}

We apply five observed wind sequences to the verified linear wind farm model and compare the performance when applying the proposed distributed controller with a controller providing static power set-points. For all five input sequences, fatigue reductions in the magnitude of $15 \%$ are observed as presented in Table I. As previously described, the variance of the tower and shaft torques are used as a measure of fatigue.

In Fig. 4 we observe a single wind turbine for one time sequence. We compare the distributed controller (blue solid curves) with the reference controller (red dashed curves). The plots illustrate that the distributed controller allows the power set-point to vary around the operating point of $2.2 \mathrm{MW}$, reducing the accumulated tower fatigue. Further we note, that the pitch is almost identical to the case of no control, indicating that the wind power plant controller only slightly changes the way each turbine operates. This indicates what has been previously stated, namely that the presented wind power plant controller will not cause significant changes in the wind fields at the downstream turbines.

\section{B. Evaluation on Industrial Model}

To further validate the distributed controller, we examine the performance using an industrial megawatt wind turbine 

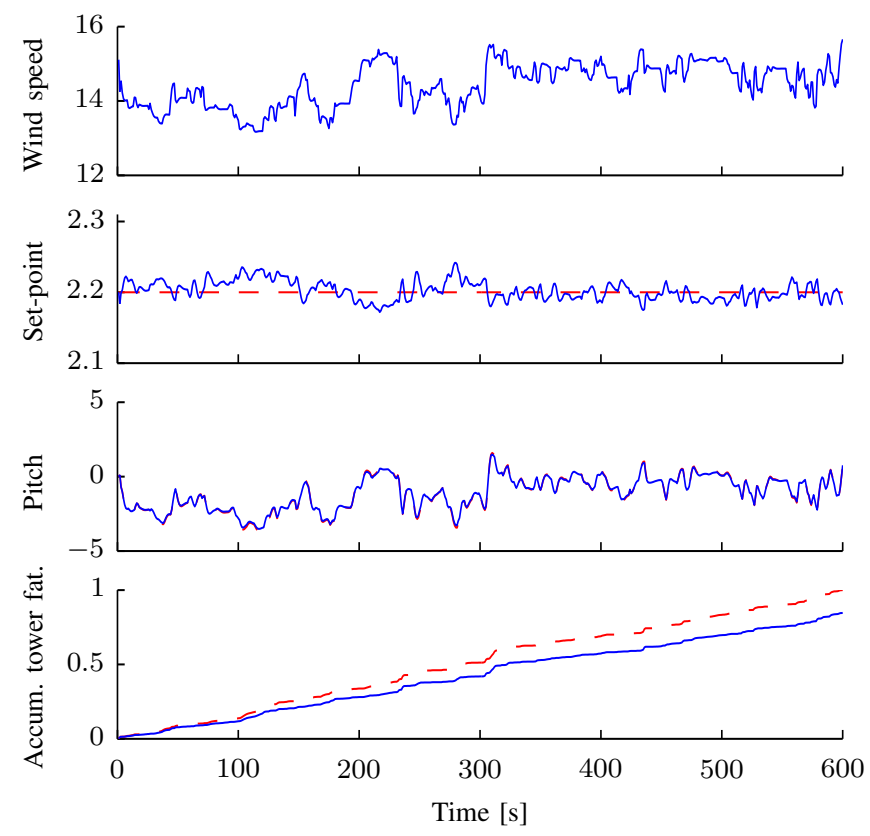

Fig. 4. Response of a single turbine based on 10 minute simulation with the reference controller (red, dotted) and with distributed wind power plant controller (blue, solid).

simulation model. This rather complex industrial model contains rate limits, measurement noise, transport delays, higher frequency responses and further non-linearities providing more reliable results.

By applying the same distributed controller as in the previous simulation, fatigue reductions as presented in Table I are obtained revealing fatigue reductions in the magnitude of $10 \%$ and $25 \%$ for tower and shaft, respectively. Further, all five simulations show that the error between the power production and the power reference does not increase with the distributed controller.

\section{CONCLUSION}

In this paper we have presented a distributed controller for wind power plants in derated operation with fatigue reduction as objective; each wind turbine updates its power set-point based on local pitch angle and generator velocity measurements and by information sharing with neighboring turbines. A major advantage of this controller is that it has very low complexity, relies on a few number measurements, and requires no filtering and only little communication, thereby making implementation simple and transparent.

We demonstrate the controller via simulations on an industrial megawatt wind power plant model applying different effective wind speed sequences obtained from an operational megawatt wind power plant. In all simulation examples, both tower and shaft fatigues are reduced by around $15 \%-20 \%$ compared to using static power set-points. These significant fatigue reductions are obtained, as the controller allows the turbines to operate according to the local wind conditions requiring only, that the total wind power plant demand is met.

\section{REFERENCES}

[1] Energinet.dk, "Smart grid in Denmark," 2011, Published on www.energinet.dk.

[2] Nord Pool, "No. 99/2009 Implementation of negative price floor in Elspot," 2009, Appeared in Market News No. 99/2009 on www.nordpoolspot.com.

[3] Danish Ministry for Climate, Energy and Buildings (Klima,- energi og bygningsministeriet), "The energy- and climate goals of the danish government and results of the energy agreement 2020 (regeringens energi- og klimapolitiske mål - og resultaterne af energiaftalen i 2020)," 2012.

[4] P. Nyeng, "System integration of distributed energy resources," Ph.D. dissertation, Technical University of Denmark, 2010.

[5] I. A. Hiskens, "Load as a controllable resource for dynamic security enhancement," in Proceedings of the IEEE Power Engineering Society General Meeting, june 2006.

[6] K. Moslehi and R. Kumar, "A reliability perspective of the smart grid," IEEE Transactions on Smart Grid, vol. 1, no. 1, pp. 57-64, 2010.

[7] K. Trangbaek, J. Bendtsen, and J. Stoustrup, Hierarchical Model Predictive Control for Plug-and-Play Resource Distribution. Springer Publishing Company, 2012, chapter 15, pages 339-358. ISBN 978-14471-2264-7.

[8] B. Biegel, J. Stoustrup, J. Bendtsen, and P. Andersen, "Model predictive control for power flows in networks with limited capacity," in Submitted for publication, 2012.

[9] P. W. Christensen and G. C. Tarnowski, "Inertia for wind power plants state of the art review year 2011," in Proceedings of 10th International Workshop on Large-Scale Integration of Wind Power into Power Systems, Aarhus, Denmark, 2011, pp. 457-463.

[10] M. Speckmann and A. Baier, "Provision of frequency control by wind farms," in Proceedings of 10th International Workshop on Large-Scale Integration of Wind Power into Power Systems, Aarhus, Denmark, 2011, pp. 470-474.

[11] D. Heide and C. H. M. Greiner, L. Bremen, "Reduced storage and balancing needs in a fully renewable european power system with excess wind and solar power generation," Renewable Energy, vol. 36, pp. 2523-2523, 2011.

[12] T. Knudsen, T. Bak, and M. Soltani, "Distributed control of largescale offshore wind farms," in European Wind Energy Conference and Exhibition (EWEC) 2009, Marseille, France, 2009.

[13] A. D. Hansen, P. Sørensen, F. Blaabjerg, and I. Florin, "Grid support of a wind farm with active stall wind turbines and ac grid connection," Wind Engineering, no. 9, pp. 341-359, 2005.

[14] B. Biegel, "Distributed control of wind farm," Master's thesis, Aalborg University, 2011.

[15] D. Madjidian, K. Mårtensson, and A. Rantzer, "A distributed power coordination scheme for fatigue load reduction in wind farms," Submitted to American Control Conference, San Francisco, June 2011.

[16] T. Burton, D. Sharpe, N. Jenkins, and E. Bossanyi, Wind Energy Handbook. Wiley, 2001.

[17] E. Muljadi and C. Butterfield, "Pitch-controlled variable-speed wind turbine generation," in Presented at the 1999 IEEE Industry Applications Society Annual Meeting, Phoenix, Arizona, 1999, pp. 470-474.

[18] T. Knudsen, T. Bak, and M. Soltani, "Prediction models for wind speed at turbine locations in a wind farm," Wind Energy, no. 14, p. 877894, 2011

[19] V. Spudić, M. Jelavić, and M. Baotić, "Wind turbine power references in coordinated control of wind farms," Automatika - Journal for Control, Measurements, Electronics, Computing and Communications, vol. 52, no. 2, pp. 82-94, April-June 2011.

[20] T. Knudsen, T. Bak, and M. Soltani, "Prediction models for wind speed at turbine locations in a wind farm," Wind Energy, vol. 14, no. 7, pp. 877-894, 2011. [Online]. Available: http://dx.doi.org/10.1002/we.491

[21] K. Hammerum, "A fatigue approach to wind turbine control," Master's thesis, Technical University of Denmark, 2006.

[22] S. Boyd and L. Vandenberghe, Convex Optimization. Cambridge University Press, 2004.

[23] T. Glad and L. Ljung, Control Theory, Multivariable and Nonlinear Methods, 1st ed. Taylor \& Francis, 2000, iSBN 0-7484-0878-9.

[24] K. Mårtensson and A. Rantzer, "Gradient methods for iterative distributed control synthesis," in Proceedings of the 48th IEEE Conference on Decision and Control, Shanghai, China, Dec. 2009. 